\title{
1 Model Generation of Coronary Artery Bifurcations from CTA and Single Plane 2 Angiography
}

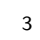

4

5

\author{
Rubén Cárdenes \\ Center for Computational Imaging $\&$ Simulation Technologies \\ in Biomedicine (CISTIB) - Universitat Pompeu Fabra and \\ Networking Biomedical Research Center on Bioengineering, \\ Biomaterials and Nanomedicine (CIBER-BBN), Barcelona, \\ Spain \\ José L. Díez \\ Cardiology Department, University Hospital Dr. Peset, Valencia, \\ Spain
}

\section{Nicolas Duchateau}

Hospital Clinic Provincial de Barcelona, Institut d'investigacions Biomèdiques August Pi i Sunyer - Universitat de Barcelona, Spain
Ali Pashaei
Center for Computational Imaging \& Simulation Technologies in Biomedicine (CISTIB) - Universitat Pompeu Fabra and Networking Biomedical Research Center on Bioengineering, Biomaterials and Nanomedicine (CIBER-BBN), Barcelona, Spain

\begin{abstract}
Alejandro F. Frangi
Center for Computational Imaging 8 Simulation Technologies in Biomedicine (CISTIB) - Universitat Pompeu Fabra and Networking Biomedical Research Center on Bioengineering, Biomaterials and Nanomedicine (CIBER-BBN), Barcelona, Spain. Dpt. of Mechanical Engineering, University of Sheffield, Sheffield, $U K$
\end{abstract}


Purpose: To generate accurate and realistic models of coronary artery bifurcations before and after percutaneous coronary intervention (PCI), using information from two image modalities. Because bifurcations are regions where atherosclerotic plaque appears frequently and intervention is more challenging, generation of such realistic models could be of high value to predict the risk of restenosis or thrombosis after stent implantation, and to study geometrical and hemodynamical changes.

Methods: Two image modalities have been employed to generate the bifurcation models: Computer Tomography Angiography (CTA) to obtain the 3D trajectory of vessels, and 2D Conventional Coronary Angiography (CCA) to obtain radius information of the vessel lumen, due to its better contrast and image resolution. In addition, CCA can be acquired right before and after the intervention in the operation room; therefore, the combination of CTA and CCA allows the generation of realistic pre and post-procedure models of coronary bifurcations. The method proposed is semi-automatic, based on landmarks manually placed on both image modalities.

Results: A comparative study of the models obtained with the proposed method with models manually obtained using only CTA, shows more reliable results when both modalities are used together. We show that using pre-procedure CTA and postprocedure CCA, realistic post-procedure models can be obtained. Analysis carried out of the Murray's law in all patient bifurcations shows the geometric improvement of PCI in our models, better than using manual models from CTA alone. An experiment using a cardiac phantom also shows the feasibility of the proposed method.

Conclusions: We have shown that fusion of CTA and CCA is feasible for realistic generation of coronary bifurcation models before and after PCI. The method proposed is efficient, and relies on minimal user interaction, and therefore is of high value to study geometric and hemodynamic changes of treated patients.

Keywords: coronary arteries, bifurcations, modeling, stenting, centerlines, fast marching 


\section{INTRODUCTION AND BACKGROUND}

Coronary artery bifurcations are regions of predominant atherosclerotic plaque formation $^{1}$, accounting for around $15 \%$ to $20 \%$ of the total amount of endovascular interventions ${ }^{2}$. Percutaneous coronary intervention (PCI) of bifurcations is particularly challenging, and a ${ }_{35}$ wide variety of techniques have been described in the last two decades ${ }^{3,4}$. There is still controversy among endovascular interventionists regarding which technique may be the most appropriate for an individual case ${ }^{5}$. Assessment of coronary artery disease (CAD) is commonly carried out using computed tomography angiography (CTA) or conventional coronary angiography $(\mathrm{CCA})^{6}$. Although CCA is still considered the gold standard due to its high40 est temporal and spatial resolution, the use of multi detector CT (MDCT) scanners has increased in the last years due to the improvement in their image quality ${ }^{7}$. CTA has overcome some of the CCAs limitations, because it allows 3D visualization of the vessels, is able to distinguish the calcified plaques, and avoids problems such as vessel overlapping or foreshortening. However, the lumen vessel radius can be better estimated from CCA due to 45 its better spatial resolution and higher intensity contrast.

If a realistic 3D geometry of the bifurcation before and after treatment is available, a detailed analysis of the vascular morphology and its changes could respond to questions such as the level of success of the treatment employed ${ }^{8,9}$ or, in a large population study, which techniques are more suitable for specific morphologies and locations. Also, computational

${ }_{50}$ fluid dynamics studies can be carried out using accurate vessel geometries ${ }^{10}$, and the study of the changes in hemodynamic parameters, could reveal regions of high risk of restenosis or thrombosis ${ }^{11}$.

Several approaches have been proposed to obtain accurate segmentations of coronary arteries. A large amount of effort has been made in the past regarding centerline tracking 55 and vessel segmentation of coronaries using CTA. A full review of the literature about these methods is out of the scope of this paper, but a comparative study of them can be found in Shaap et al. ${ }^{12}$ Other segmentation approaches have been recently proposed using multi-view variants of conventional coronary angiography (CCA). For instance, biplane angiography ${ }^{13,14}$ or 3D rotational angiography (3DRA $)^{15,16}$, have been used to obtain 3D coronary models.

${ }_{60}$ These approaches require sophisticated methods for reconstruction including gating and motion compensation techniques to account for heart and respiratory motion. All these 
methods are based on the information provided by a single modality, either CTA, 3DCA or biplane CCA.

In this paper, we propose to combine two modalities: CTA and single plane CCA, taking ${ }_{65}$ advantage of each one to overcome the limitations of the other. Therefore, realistic 3D bifurcation geometries can be obtained using routinely acquired imaging techniques, with a semi-automatic method that provides the pre and post-procedure models of the vessels. The method proposed here, provides a formal description and validation of a work presented before $^{17}$, by comparing our models with CTA alone, with CCA alone, and using a phantom 70 device to show the feasibility of the method. This is, as far as we know, the first time that these two modalities are combined for the reconstruction of coronary artery bifurcations.

\section{METHODS AND MATERIALS}

\section{A. Semi-automatic model generation from CTA and CCA}

The method proposed to generate models from these two modalities essentially consists of 75 three steps. First, the 3D structure of the bifurcation is obtained from CTA pre-procedure data using a ridge extraction method ${ }^{18}$ followed by a fast marching (FM) approach to obtain a connected vessel centerline. After that, CCA images obtained before and after endovascular treatment are processed to obtain the vessel radii along the vessel centerlines, and finally, the models are constructed mapping the vessel radii to the 3D centerline structure.

\section{1. 3D centerline extraction}

In the first stage of the method, CTA images are used to extract the vessel centerlines around the bifurcation under study. In this step, the user specifies the center of the bifurcation in the CTA image, that will be denoted as $\boldsymbol{b}^{3 \mathrm{D}}$, and a minimum of three points on the vessels forming the bifurcation, denoted as $\boldsymbol{p}_{1}^{3 \mathrm{D}}$ for the proximal segment of the princi${ }_{85} \mathrm{pal}$ branch, $\boldsymbol{p}_{2}^{3 \mathrm{D}}$, for the distal segment of the principal branch, and $\boldsymbol{p}_{3}^{3 \mathrm{D}}$ for the secondary branch. Hereafter, the subindices $i=\{1,2,3\}$ will denote these three branches in this order. Then, the ridge extraction algorithm ${ }^{18}$, available within the open-source TubeTK toolkit ${ }^{19}$ is employed on the image using $\boldsymbol{p}_{1}^{3 \mathrm{D}}, \boldsymbol{p}_{2}^{3 \mathrm{D}}$, and $\boldsymbol{p}_{3}^{3 \mathrm{D}}$ as seeds, to extract the centerlines. To obtain better results, the vessel enhancing filtering by Manniessing et al. ${ }^{20}$ is applied to the 

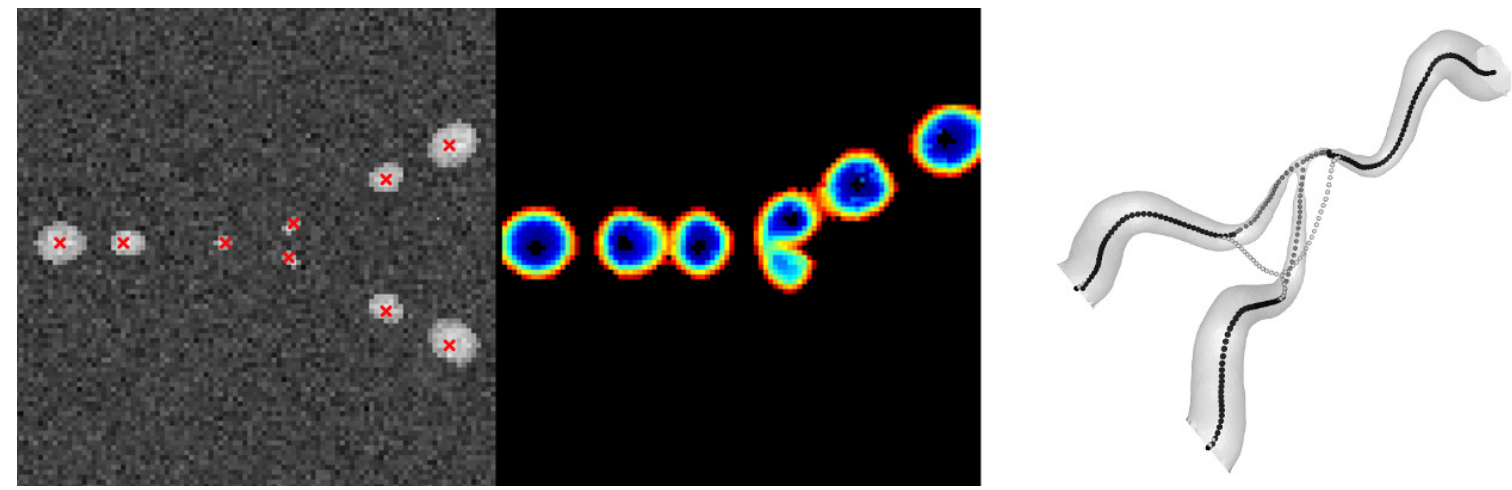

FIG. 1. Slice of a digital phantom with centerline points overlaid (left), same slice with $u(\boldsymbol{x})$ computed from one segment (middle), and 3D view of the digital phantom (right), with initial centerline points in blue, connected points with our proposed speed function in red, and connected points using constant speed in green.

${ }_{90}$ CTA image prior to the ridge extraction.

The centerlines obtained from the ridge extraction process do not always form a connected set. Discontinuities in the centerlines appear due to several reasons, such as the relatively low spatial and temporal resolution of the CTA image, noise, low contrast, and especially due to lesions affecting the bifurcation. For this reason, a reconnection strategy is ${ }_{95}$ employed similarly to Mueller et al. ${ }^{21}$ The reconnection method consists in finding for each disconnected segment, the closest point in any of the other segments, $\boldsymbol{y}$, using a distance map $u(\boldsymbol{x})$. Then, a minimum cost path is computed from $\boldsymbol{y}$ to the initial segment using $u(\boldsymbol{x})$. To compute $u(\boldsymbol{x})$ a FM algorithm is employed, solving the Eikonal equation:

$$
|\nabla u(\boldsymbol{x})| F(\boldsymbol{x})=1,
$$

and using the disconnected segment points as seeds. The FM computation is stopped when 100 one point $\boldsymbol{y}$ in any of the other segments is reached. Here, the speed function $F(\boldsymbol{x})$ has to be carefully selected. A constant speed function will provide an Euclidean distance map, that will connect the vessel centerlines by straight lines, thus, providing non realistic results (see green points in Fig. 1). In order to follow a more realistic trajectory of the vessels, a speed function that depends exponentially (as in Hassouna et $a .^{22}$ ) on the local vessel structure 105 is used as follows:

$$
F(\boldsymbol{x})=e^{\alpha \cdot V(\boldsymbol{x}) / M},
$$


where $V(\boldsymbol{x})$ is the multi-scale vesselness of the $\mathrm{CTA}^{23}$, and $M$ is the maximum value in $V(\boldsymbol{x})$. Ten scales are used to compute $V(\boldsymbol{x})$, from 0.2 up to $4 \mathrm{~mm} . \quad F(\boldsymbol{x})$ is designed to drive the FM process faster along vessels and the parameter $\alpha$ controls its strength. Low $\alpha$ values give a speed closer to constant everywhere, and high values increment the speed 110 at regions with high vesselness values. Here, the value used is $\alpha=2$ that provides good results experimentally. The reconnection process is repeated for each segment until there is only one connected segment. The behavior of this reconnection strategy is illustrated in the digital phantom shown in Fig. 1, where three disconnected segments are connected following the tubular structures. This phantom has added Gaussian noise with zero mean, providing a 115 signal to noise ratio of 12 . In this figure, an orthogonal slice of the phantom with centerline points overlaid is shown (left), as well as the distance map $u(\boldsymbol{x})$, computed from one segment (middle), and the resulting centerline (right), with the connection points obtained using the speed function proposed (red) and those obtained using a constant speed function (green). Note how the connection points using a speed function based on the vesselness follow the 120 tubular structure, whereas using a constant speed function results in straight connections disregarding the tubular shape of the phantom. In Fig. 2 the centerline points obtained from the ridge extraction (red points) and reconnection step (green points) are shown for a left coronary artery tree.

The ridge extraction algorithm follows the high intensity values in the image, correspond125 ing to contrast agent present in the vessels. However, the calcified tissues of the plaque, if present, are also visible in CTA, but are not part of the vessel lumen, and therefore have to be removed prior to the centerline extraction. For this reason, calcifications are subtracted from the CTA image before the ridge extraction step. This can be done by thresholding, using a standard threshold of 721 Hounsfield units as described by Sakakura et al. ${ }^{24}$. Using 130 the Hounsfield scale assures that the threshold value will be consistent across all the CTA scans used. Fig. 2 (left) shows a CTA slice of one real case with two different centerlines, one computed using the CTA image without calcium removed (red points), where the centerline follows the high intensity values, and the other using the CTA with the calcium removed (green points), where the centerline follows the vessel lumen better, avoiding the plaque. 

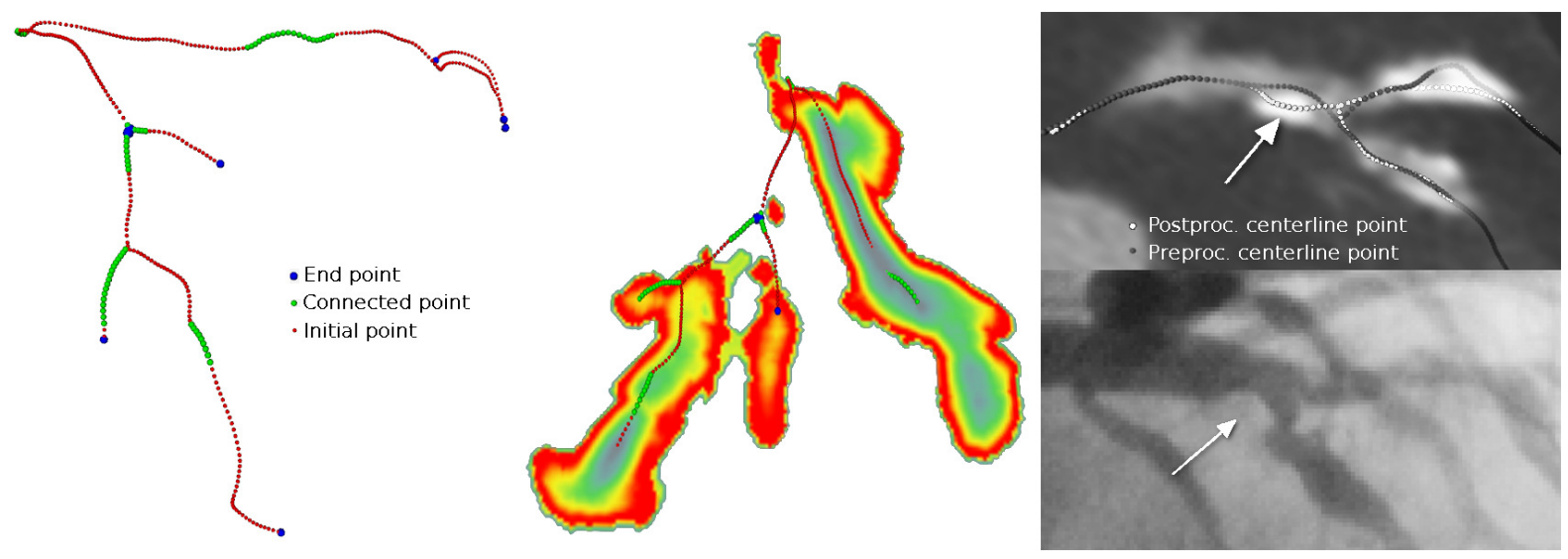

FIG. 2. Left: Initial centerline points (red) obtained from CTA, connected centerline points using FM (green) and end points and bifurcation points (blue). Middle: slice of the distance field $u(\boldsymbol{x})$ from one segment. Right top: centerlines of the post-procedure model (red), and pre-procedure centerline (green) avoiding the calcified plaque pointed by arrows. Right bottom: corresponding CCA image.

\section{2. Processing of the 2D angiographic images}

In the following step, the vessel radii on the CCA images are calculated. In particular, two images are selected from pre and post-procedure views, using an angle where the area of the projection of the bifurcation is maximum. This projection corresponds approximately to the CCA image chosen by the clinician for an optimal visualization of the bifurcation,

140 minimizing foreshortening and vessel overlap. Notice that only vessel radii and lengths from the bifurcation center are used from CCA, and therefore there is no need to have a perfect projection matching between CTA and CCA. This is especially important because the vessel geometry extracted from CTA can vary with respect to CCA obtained when the guide wire is inserted, changing the vessels curvature (see Fig. 3). Therefore, our method does not 145 need perfect correspondence between CTA and CCA, being robust with respect to geometry changes and with respect to the cardiac phase used in both modalities. One alternative method could be to perform an elastic 2D registration between CCA and CTA projections to find radii correspondences, but the lack of information about the projection geometry of the CCA acquisition would increase considerably the complexity of the problem as well as 150 the computational cost.

For each of the two projection images selected, the bifurcation center, denoted as $\boldsymbol{b}^{2 \mathrm{D}}$, 


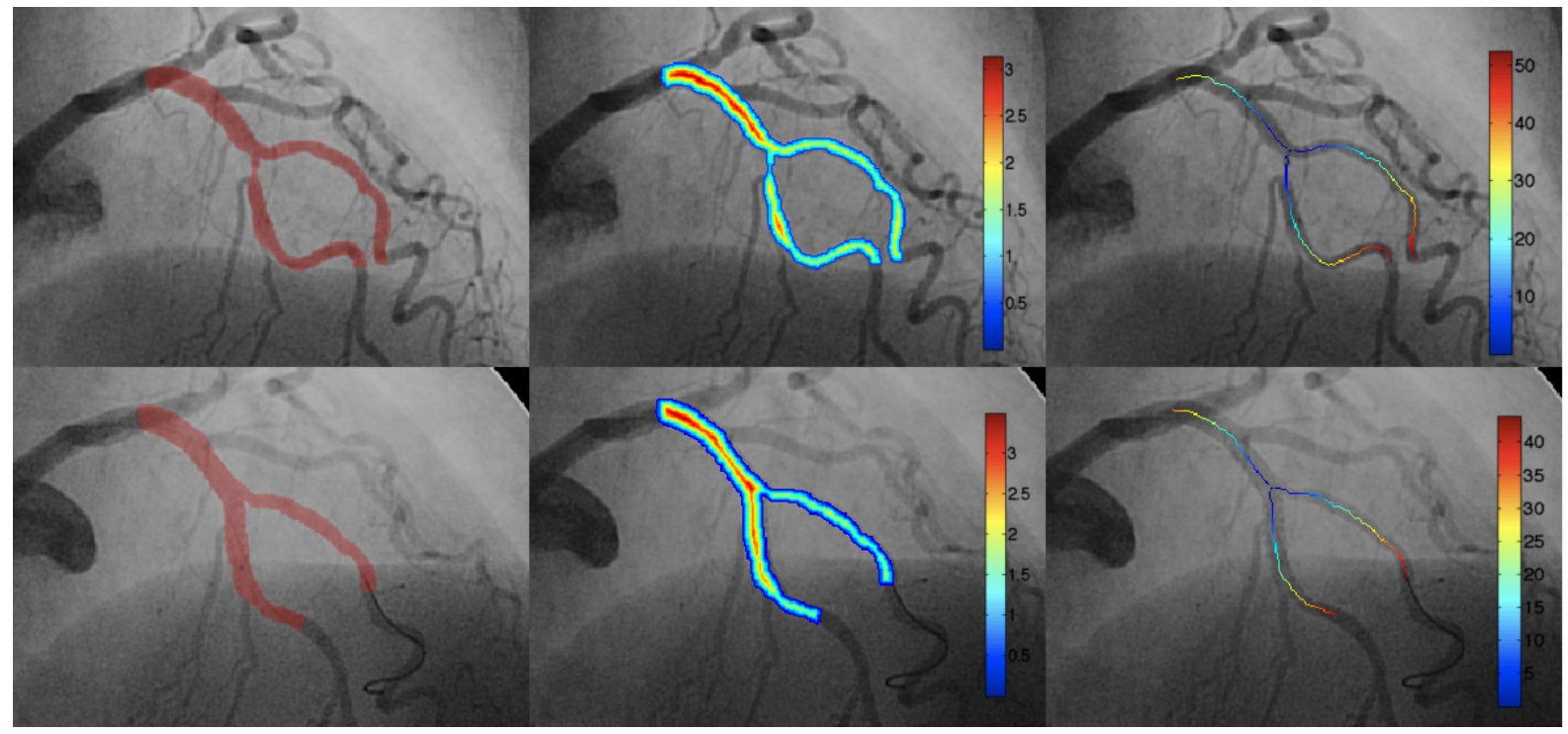

FIG. 3. CCA images of a treated patient. Top row: pre-procedure, bottom row: post-procedure. Overlay images are from left to right: segmentation, distances from the vessel boundary, and centerline branches colored according to the the lengths from $\boldsymbol{b}^{2 \mathrm{D}}$.

is also manually marked, which will correspond to the point $\boldsymbol{b}^{3 \mathrm{D}}$. Also, three points on the branches are marked $\left(\boldsymbol{p}_{1}^{2 \mathrm{D}}, \boldsymbol{p}_{2}^{2 \mathrm{D}}\right.$ and $\left.\boldsymbol{p}_{3}^{2 \mathrm{D}}\right)$, which will be used to assign correspondences between the $2 \mathrm{D}$ and $3 \mathrm{D}$ centerlines and to stop the $2 \mathrm{D}$ segmentation process, but do not 155 have to correspond accurately to $\boldsymbol{p}_{i}^{3 \mathrm{D}}$. The vessel lumen in the CCA images are segmented using a level set approach starting from $\boldsymbol{b}^{2 \mathrm{D}}$ and stopping when the user defined points $\boldsymbol{p}_{i}^{2 \mathrm{D}}$ are reached. Then, the vessel centerlines are extracted, and finally, the vessel radii are computed as the distances from the centerline points to the closest segmented boundary. Due to the cone beam X-ray image formation, the angiography images are magnified by a 160 factor of $D / d$, with $D$ the distance from source to detector and $d$ the distance from source to object. Therefore, a correction factor $d / D$ has to be applied to the measures performed on the angiographic images. Because $D$ can be accurately known but $d$ can vary depending on the data, the correction factor was derived experimentally from the data, measuring the known lengths of the catheter tip on the X-ray images, and giving an average value of 165 0.6. This value was used for all the real cases studied here. Two pre and post-procedure CCA images are shown in Fig. 3 with the segmentation, the distances from the segmented boundaries and the centerlines overlaid. 


\section{Radius mapping}

In the next step the radii values computed in the $2 \mathrm{D}$ angiographic images are mapped to 170 the $3 \mathrm{D}$ centerlines, to construct the bifurcation models. In this work, circular cross-section is assumed to construct the models, which is true in healthy and treated arteries, and in $70 \%$ to $80 \%$ of the lesions, according to the study performed by Arbab-Zadeh et al. ${ }^{6}$

The radius mapping is implemented using the centerline lengths. Let $\Gamma_{i}(s)$ and $\gamma_{i}(s)$ be the parameterized centerline curves of each bifurcation branch in 3D and 2D respectively, ${ }_{175}$ where $\Gamma_{i}(0)=\boldsymbol{b}^{3 \mathrm{D}}, \Gamma_{i}(1)=\boldsymbol{p}_{i}^{3 \mathrm{D}}$ and $\gamma_{i}(0)=\boldsymbol{b}^{2 \mathrm{D}}$. Then, we choose $\gamma_{i}(1)$ as the $2 \mathrm{D}$ point such as corresponding curves have the same length, $L\left(\Gamma_{i}(1)\right)=L\left(\gamma_{i}(1)\right)=L_{i}$, where the length function $L_{i}(\gamma(s))$ is given by

$$
L_{i}(\gamma(s))=\int_{0}^{s}\left\|\gamma_{i}^{\prime}(t)\right\| d t
$$

Then, a radius value is assigned to each point on $\Gamma_{i}$ defining a radius mapping function dependent on the curve length,

$$
R_{i}:\left[0, L_{i}\right] \rightarrow \mathbb{R}^{+}, i=\{1,2,3\}
$$

This function maps the radius value obtained at each point of the $\mathrm{i}^{\text {th }} 2 \mathrm{D}$ branch to the corresponding point in the $\mathrm{i}^{\text {th }} 3 \mathrm{D}$ branch, where the correspondence between the $3 \mathrm{D}$ point ${ }_{180} \Gamma_{i}(s)$, and the $2 \mathrm{D}$ point $\gamma_{i}(s)$, is obtained by the length with respect to the bifurcation point. In other words, if the length from $\Gamma_{i}(s)$ to $\boldsymbol{b}^{3 \mathrm{D}}$ is equal to the length from $\gamma_{i}(s)$ to $\boldsymbol{b}^{2 \mathrm{D}}$, the radius obtained for $\gamma_{i}(s)$, will be assigned to $\Gamma_{i}(s)$. Denoting the radius of a point in the $i^{\text {th }}$ $2 \mathrm{D}$ branch as $r_{i}\left(L\left(\Gamma_{i}(s)\right)\right)$, then the radius mapping function is given by:

$$
R_{i}\left(L\left(\Gamma_{i}(s)\right)\right)=r_{i}\left(L\left(\gamma_{i}(s)\right)\right), i=\{1,2,3\}
$$

In general, there is no exact correspondence between the points $\Gamma_{i}(s)$ and $\gamma_{i}(s)$ due to 185 foreshortening. However, we can assume that in a small region near $\boldsymbol{b}^{3 \mathrm{D}}$, the centerlines of the bifurcation branches are nearly co-planar ${ }^{25}$, and thus, the foreshortening effect is minimum on this plane, and the radius mapping can be applied. Finally, the obtained surfaces are smoothed to avoid sharp transitions at vessel junctions. 


\section{Predictive post-procedure models}

190 CTA and post-procedure CCA. This option combines the 3D trajectory information from pre-procedure CTA and maps the radius values of post-procedure CCA, assuming that the vessel trajectories do not change considerably after stenting. This assumption does not always hold because the vessels may be straightened after PCI, however, this effect 195 was observed to be low. The centerlines used for these models were computed from the preCTA images without the calcium removal step described before, to obtain centerlines following a trajectory without deviations from the calcified plaques. This will give more similar centerlines to the actual post-procedure trajectories. These models will be denoted as predictive post-procedure models or preCTA+postCCA models.

\section{5. Limitations of the method}

Two main assumptions have been used in this methodology. First, we assume circular cross section of the vessels, which is true for healthy and treated vessels, and therefore will be valid in post-operative models, and, as mentioned above, it is also valid in $70 \%$ to $80 \%$ of the lesions as stated in the literature ${ }^{6}$. A second assumption is planar correspondence between 205 the CCAs and the bifurcation vessels in a small region around its center. Therefore, it is crucial to make a good choice of the CCA images, in order to avoid foreshortening; however, small deviations from the selection of this plane do not affect substantially the models as we will show in the experiment carried out with a phantom. Regarding CCA images, it is important to highlight that a magnification correction factor has to be applied to measure 210 lengths and radii, but it can be known using a reference object with known length as the catheter tip.

Regarding the reconnection strategy proposed in the centerline extraction method, we observed its robustness, but the procedure can fail to connect two centerline segments if they are too far away from each other, or if another bifurcation is present in the middle. ${ }_{215}$ However, this can be corrected by adding new landmarks or changing the initial ones. In our cases, only one landmark per branch was needed to obtain connected centerlines of the bifurcations. 
A final assumption made when using preCTA+postCCA data to obtain post-procedure models is that the vessel trajectories do not change after PCI, which is not always true be220 cause PCI can modify the vessel curvature and the bifurcation angles, although the changes observed are small, as we will show in the results.

\section{B. Manual model generation from CTA}

To test the models obtained with our methodology with respect to the information given by CTA data alone, a manual model generation using CTA data has been used. This process 225 was performed manually to obtain models resembling as much as possible the geometry provided by CTA images. However, an automatic initialization was done to guide the user in this difficult task, and to minimize errors due to user fatigue. The initialization was performed by radius estimation of the vessels from the centerlines obtained in section II A 1 , although any other initial segmentation method could have been used. Manual correction 230 was performed using the intensity values from CTA, with the itk-SNAP v2.0 open source software $^{26}$. The user could change the intensity level and window until he/she is satisfied, and those values were kept during the rest of the segmentation correction. The corrections were performed by the user through region selection on a viewer with a mouse showing grayscale $2 \mathrm{D}$ orthogonal views of the CTA data with the segmentation overlaid on top. It is important 235 to highlight that manual segmentations without initialization appeared unrealistic, due to contrast changes along a big number of slices. Finally, the models were smoothed to remove sharp transitions.

\section{Materials}

\section{Patient data}

Five patients have been selected for this study, all of them affected by a stenosis in a coronary bifurcation and eligible for PCI, and who voluntarily signed informed consent for this study. The lesions treated were located at the main bifurcation of the left anterior descending (LAD) artery bifurcation for two cases, and at bifurcations of the proximal and medial segments of the LAD, (LADp, LADm), for the rest of cases. They were treated using 245 several techniques, provisional T-stenting (three cases), provisional T-stenting with final 
kissing balloon (one case), and crushing (one case). All the patients had a pre-procedure CTA scan, which was acquired in a 64 multi-slice scanner, LightSpeed VCT, from GE Medical Systems, with an image resolution of $0.445 \times 0.445 \times 0.625 \mathrm{~mm}^{3}$. For each patient, two CCA 2D image projections (pre and post-stenting) corresponding to the same projection 250 angle and the same cardiac phase, were selected, where the bifurcation and stenosis were well seen and there was no vessel overlap. The image pixel size for those images was 0.22 $\times 0.22 \mathrm{~mm}^{2}$, with a magnification correction factor of 0.6 . Three patients had a new CTA scan 6 months after the intervention. Table I summarizes the main information for all the patients. Patient \#4 had two bifurcations involved, at LADp and LADm, therefore is was 255 necessary to perform the radius mapping twice, one for each bifurcation, and then merge the two bifurcation models obtained.

TABLE I. Summary of patient data. P. T-Stent: Provisional T-Stenting. P. T-Stent wFK: Provisional T-Stenting with final kissing.

\begin{tabular}{ccccc}
\hline Patient \# & Lesion & Technique & Stents & Follow-up CTA \\
\hline 1 & LM-LAD & P. T-Stent wFK & 1 & Yes \\
2 & LM-LAD & Crushing & 1 & Yes \\
3 & LADp & P. T-Stent & 1 & Yes \\
4 & LADp, LADm & P. T-Stent & 2 & No \\
5 & LADm & P. T-Stent & 1 & No
\end{tabular}

\section{Heart phantom}

A phantom experiment has been performed using a dynamic multi-modality heart phantom (DHP-01, Shelley Medical Imaging Technologies, London, ON, CA) to show the fea260 sibility of the proposed method. The heart phantom was wired with plastic/silicon tubes around it, resembling the coronary arteries, as shown in Fig. 4 a). Two different acquisitions were performed: a CT scan (SOMATOM Definition Flash, Siemens, Erlangen, DE) and an X-ray angiography with a C-arm device, (Arcadis Avantic, Siemens, Erlangen, DE). For the CT scan, the tubes were completely filled with water to prevent artifacts due to the presence 265 of air or contrast agent. The volume obtained was $512 \times 512 \times 230$ in size with voxel size 
of $0.262 \times 0.262 \times 0.6 \mathrm{~mm}^{3}$, a similar voxel size as the CT images for patients used in this work.

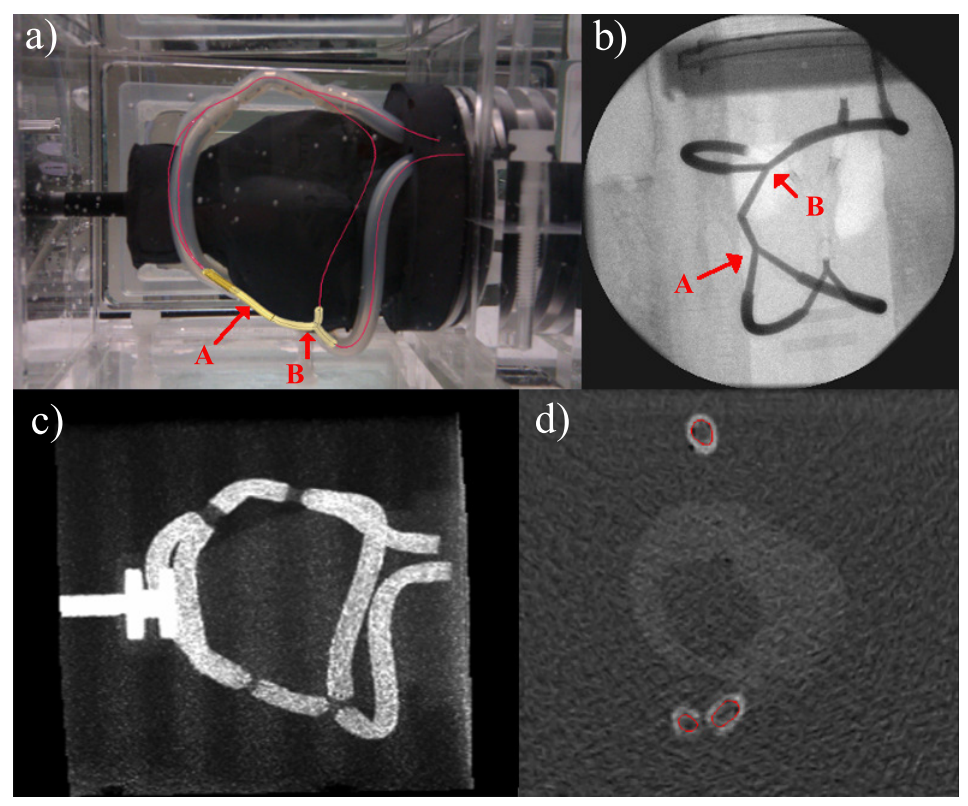

FIG. 4. Phantom used for the experiment, with tube centerlines and ground truth models overlaid, a); angiography image at 0 degrees, b); 3D volume rendering of the CT data, c); CT slice with tube segmentation overlaid, d).

For the X-ray angiography scans, the tubes were partially filled with contrast agent (iodine) resulting in an image quality very similar to that obtained for patients. In these 270 scans, two different bifurcations where clearly seen without overlapping, which we will denote as bifurcations $\mathrm{A}$ and $\mathrm{B}$, as marked in Fig. $4 \mathrm{~b}$ ). Four 2D projections were taken changing the acquisition angle from 0 to 15 degrees every 5 degrees, in order to study the differences induced in the models due to angle changes and foreshortening. The projection at 0 degrees was chosen to be approximately parallel to bifurcation A. Both acquisitions were performed 275 with the phantom in static state. A 3D volume rendering of the CT is shown in Fig. 4 c), and one CT slice is also shown in Fig. $4 \mathrm{~d}$ ).

The methodology to obtain the 3D bifurcation models from both modalities was the same as described before, and was applied to the two bifurcations considered, with maximum branch length of $18 \mathrm{~mm}$. The same magnification correction factor was applied to all 280 measurements performed on the X-ray angiographic images. This factor was estimated from several manual measurements of the tube diameter at 12 different locations of the baseline 
angiography (at 0 degrees), and averaged afterwards. The ground truth models for both bifurcations were obtained by first computing the centerlines from the CT volume, and then constructing a tube of constant diameter, using the known interior tube radius of $1.75 \mathrm{~mm}$.

${ }_{285}$ The interior radius of the tubes becomes wider at $18 \mathrm{~mm}$ from the bifurcation, but this part was not considered in our study for being far from the bifurcation plane.

\section{RESULTS}

\section{A. Results of semi-automatic models generated from CTA and CCA}
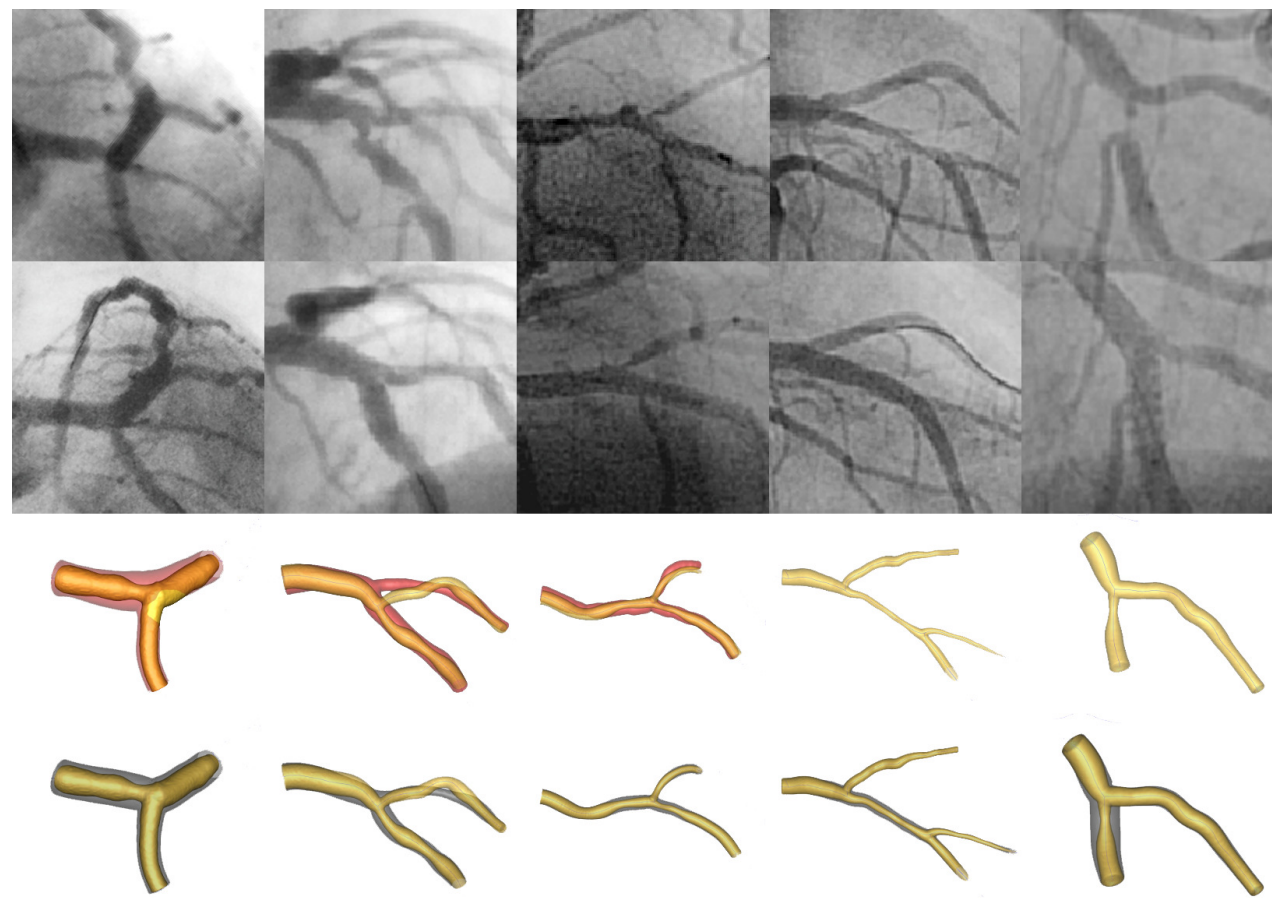

FIG. 5. Detail of the pre (first row) and post-procedure CCAs (second row), used to construct the vessel models (last two rows) in the five patients studied; in yellow preCTA+preCCA models, in red postCTA+postCCA models, in gray preCTA+postCCA models.

Fig. 5 shows the models generated using our method of fusion of CTA and CCA for 290 both, pre and post-procedure cases. To simplify, we will refer to them as semi-automatic preCTA+preCCA, or postCTA+postCCA models. The corresponding regions of the pre and post-procedure CCAs used to generate the models are also shown, to illustrate visually the information given by CCA images. It can be seen from the models the main differences 
at the stenosis regions, before and after the stenting, and their relation with the CCAs. The 295 predictive post-procedure models generated using preCTA and postCCA are also shown in the last row of Fig. 5 (in gray).

\section{B. Phantom results}

To measure the accuracy of the obtained models, two different quantities were computed. First, an overlap index, the Dice similarity coefficient DS or Sorensen index ${ }^{27}$, was computed 300 between the obtained models and the ground truth models in a volume based approach. DS is defined as $2|x \cap y| /(|x|+|y|)$, where $x$ and $y$ are regions belonging to the interior of the vessels, and $|x|$ stands for the number of elements of $x$. Second, the distances from each surface model to the ground truth surface model were also computed. The results obtained are summarized in table II, and in Fig. 6, the bifurcations models are shown with 305 colors representing the distances values to the ground truth. The maximum distance value obtained among all models was $0.42 \mathrm{~mm}$. The DS values of bifurcation A shown in table II slightly decrease (and the average distances increase) from $0^{\circ}$ to $15^{\circ}$, but not in bifurcation $\mathrm{B}$ that has a maximum of DS and a minimum of average distances at $10^{\circ}$, corresponding to the angle where this bifurcation is more parallel to the acquisition plane.

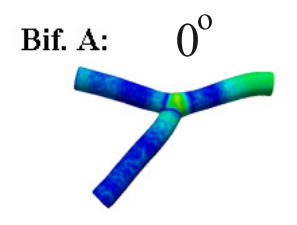

Bif. B:
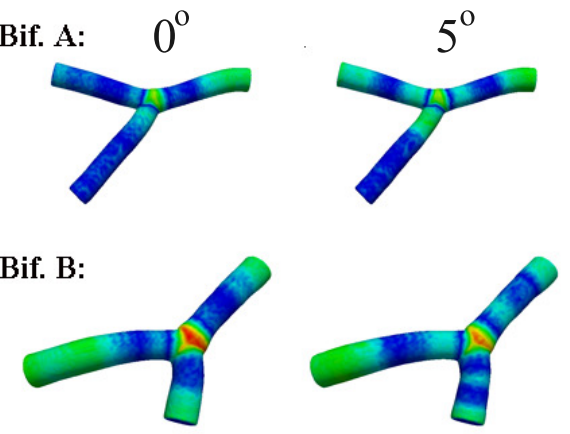
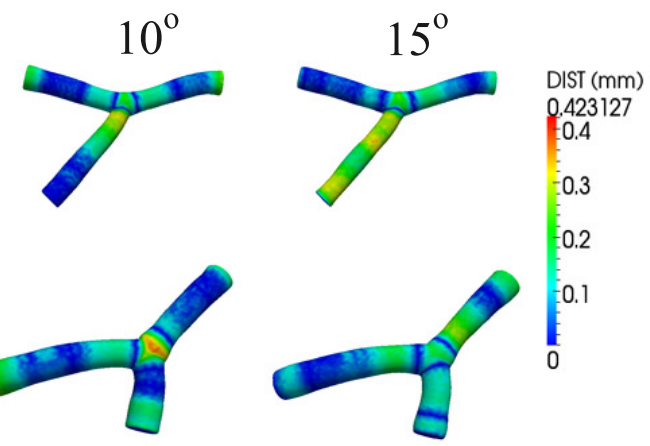

FIG. 6. Surface models obtained from the phantom at 4 different angle acquisitions with our method, colored by the distance to the ground truth. 
TABLE II. Dice similarity index and average distances computed between 3D semi-automatic models generated using $\mathrm{CT}+\mathrm{CCA}$ and $3 \mathrm{D}$ ground truth models of the phantom for two different bifurcations at 4 different angle positions of the angiography.

\begin{tabular}{cccccc}
\hline Degrees: & 0 & 5 & 10 & 15 \\
\hline \hline DS & Bif A : & 0.964 & 0.954 & 0.947 & 0.926 \\
& Bif B : & 0.940 & 0.945 & 0.949 & 0.943 \\
\hline Avg Dist & Bif A : & 0.061 & 0.078 & 0.088 & 0.118 \\
$(\mathrm{~mm})$ & Bif B : & 0.106 & 0.095 & 0.087 & 0.093 \\
\hline
\end{tabular}

${ }_{310}$ C. Model Comparison with CTA manual data

The models obtained with our method using the two modalities have been compared numerically with respect to models manually generated from CTA, as described in section II B. The differences between models obtained with this manual approach and models obtained using our semi-automatic fusion approach can be visually appreciated for each case in Fig. 7, 315 for the pre-procedure cases and in Fig. 8 for the post-procedure cases.

It is clear from Fig. 7 and Fig. 8 (top row) that models obtained from CTA alone, are in general wider, tending to overestimate the vessel lumen. This can be explained by the lower image resolution of CTA. Table III shows the Dice Similarity (DS) indices and average distance values between the models semi-automatically generated using two 320 modalities against models manually generated using only CTA. For the DS computation, all the models were voxelized at $0.1 \mathrm{~mm}^{3}$ isotropic voxel size, and the distance computation was performed on the mesh elements of the surface models. The DS indices are higher and distances are lower when the models are constructed using full pre and post-procedure, indicating reasonable similarity between them. The preCTA+postCCA models show lower ${ }_{325}$ DS and higher distances, when comparing them to post-CTA manual models. There are several reasons for this: first, the two models were generated using different 3D centerlines (one from preCTA and the other from postCTA), therefore, there are some misalignments derived from vessel changes after treatment, such as vessel curvature, and bifurcation angles, and also misalignments due to the extraction of centerlines at two different image acquisitions 330 separated by 6 months in time, that although from the same machine, are not necessarily the same image quality. Another source of error is due to the difference between our automatic 
radius mapping and the manual segmentation.
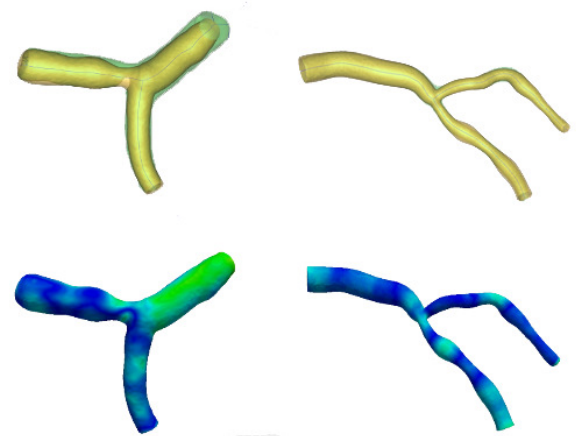
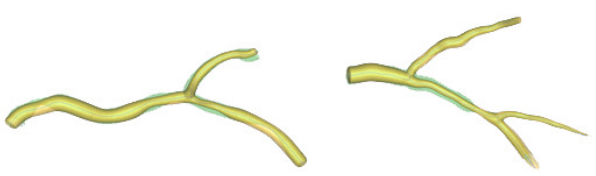
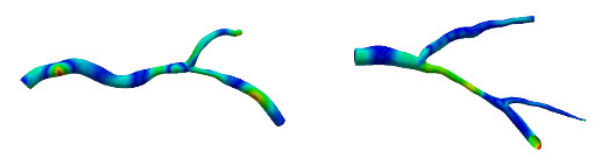
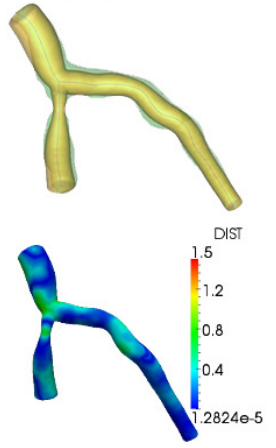

FIG. 7. Top row: pre-procedure models semi-automatically generated using preCTA+preCCA (yellow) and manually generated using preCTA alone (green) in the five patients studied. Bottom row: corresponding semi-automatic preCTA+preCCA models with colors representing the distances to the preCTA manual models.
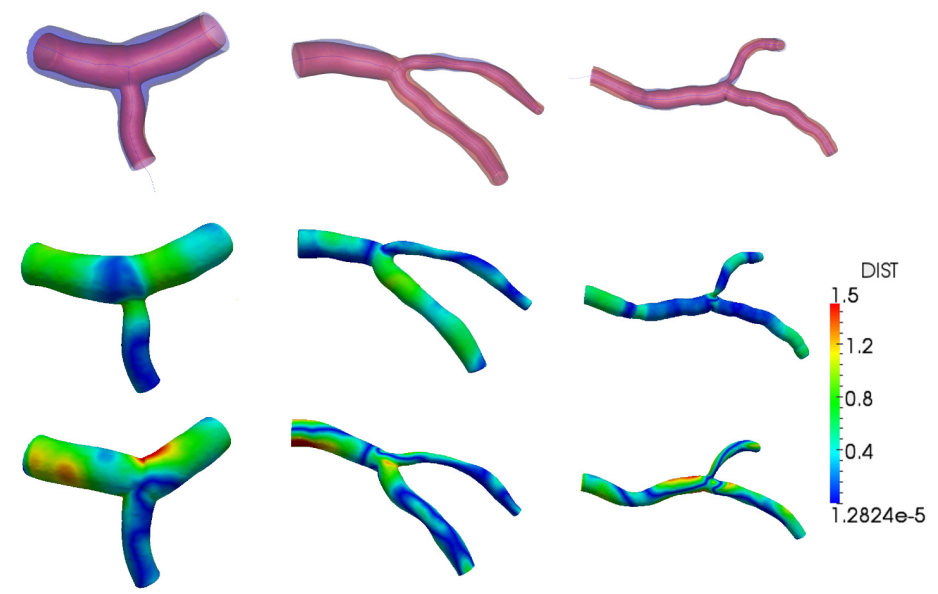

FIG. 8. Top row: semi-automatic models generated using postCTA+postCCA (red) and manual models from postCTA alone (blue). Middle row: semi-automatic postCTA+postCCA models with colors representing the distances to the postCTA manual models. Bottom row: semi-automatic preCTA+postCCA models with colors representing the distances to the postCTA manual models. These models correspond to patients \#1, \#2 and \#3 
TABLE III. Dice similarity index and average distances (in $\mathrm{mm}$ ) computed between 3D semiautomatic models generated using CTA+CCA and manual models generated using CTA alone for pre and post-procedure cases and for the 5 patients studied.

\begin{tabular}{|c|c|c|c|c|c|c|c|}
\hline \multicolumn{2}{|l|}{ Patient \# } & 1 & 2 & 3 & 4 & 5 & average \\
\hline Semi-automatic preCTA+preCCA & DS & 0.860 & 0.837 & 0.751 & 0.744 & 0.779 & 0.794 \\
\hline vs manual preCTA & avg dist & 0.275 & 0.188 & 0.358 & 0.320 & 0.253 & 0.279 \\
\hline Semi-automatic postCTA + postCCA & $\mathrm{DS}$ & 0.764 & 0.729 & 0.801 & NA & NA & 0.764 \\
\hline vs manual postCTA & avg dist & 0.498 & 0.330 & 0.270 & NA & NA & 0.366 \\
\hline Semi-automatic preCTA+postCCA & $\mathrm{DS}$ & 0.661 & 0.627 & 0.665 & NA & NA & 0.651 \\
\hline vs manual postCTA & avg dist & 0.503 & 0.493 & 0.539 & NA & NA & 0.512 \\
\hline
\end{tabular}

\section{Models comparison with angiography data}

In this section, semi-automatic models obtained from fusion of modalities and manual 335 models obtained from CTA are compared with CCA data. Due to the 2D nature of the CCA images, this comparison has been performed measuring the radii values in the models and in the CCA images along each branch, considering these latter ones the ground truth. The average values of the radii obtained from CCA in all patients studied are listed in table IV for reference. Fig. 9 shows the absolute value of the radii differences, in $\mathrm{mm}$, for each branch 340 studied, in all the generated models, taking the radius values from the CCA images as the reference. Each radius difference shown is computed as the average value of all the radius differences along the vessel branch, using radii profiles to capture local differences along the vessel. The standard deviation obtained for each vessel branch is also shown in the figure. Fig. 9 (bottom right) shows the radii profiles along one branch centerline from two different 345 models in one real case, and the radius profile obtained from the corresponding CCA image. In this figure, we denote as prox to the proximal segment of the principal branch, dist to the distal segment of the principal branch and $s e c$ to the secondary branch. From this figure, it can be noticed that distance values of CTA+CCA models (top row) are low, as we might expect because these models have been generated using the radii obtained from CCA. 350 However, these values are not zero due to model generation processes and smoothing, with values up to $0.13 \mathrm{~mm}$, but in average of $0.06 \mathrm{~mm}$. If relative errors are measured using the 
averaged radius along each branch and CCA radii as the reference, the average value is $5 \%$ and the maximum is around $10 \%$. Notice that the differences obtained from manual models obtained using CTA alone are much higher in general, with average differences, maximum 355 difference and standard deviation of $0.30 \mathrm{~mm} / 0.61 \mathrm{~mm} / 0.19 \mathrm{~mm}$, compared to $0.06 \mathrm{~mm} /$ $0.13 \mathrm{~mm} / 0.07 \mathrm{~mm}$ for semi-automatic models.
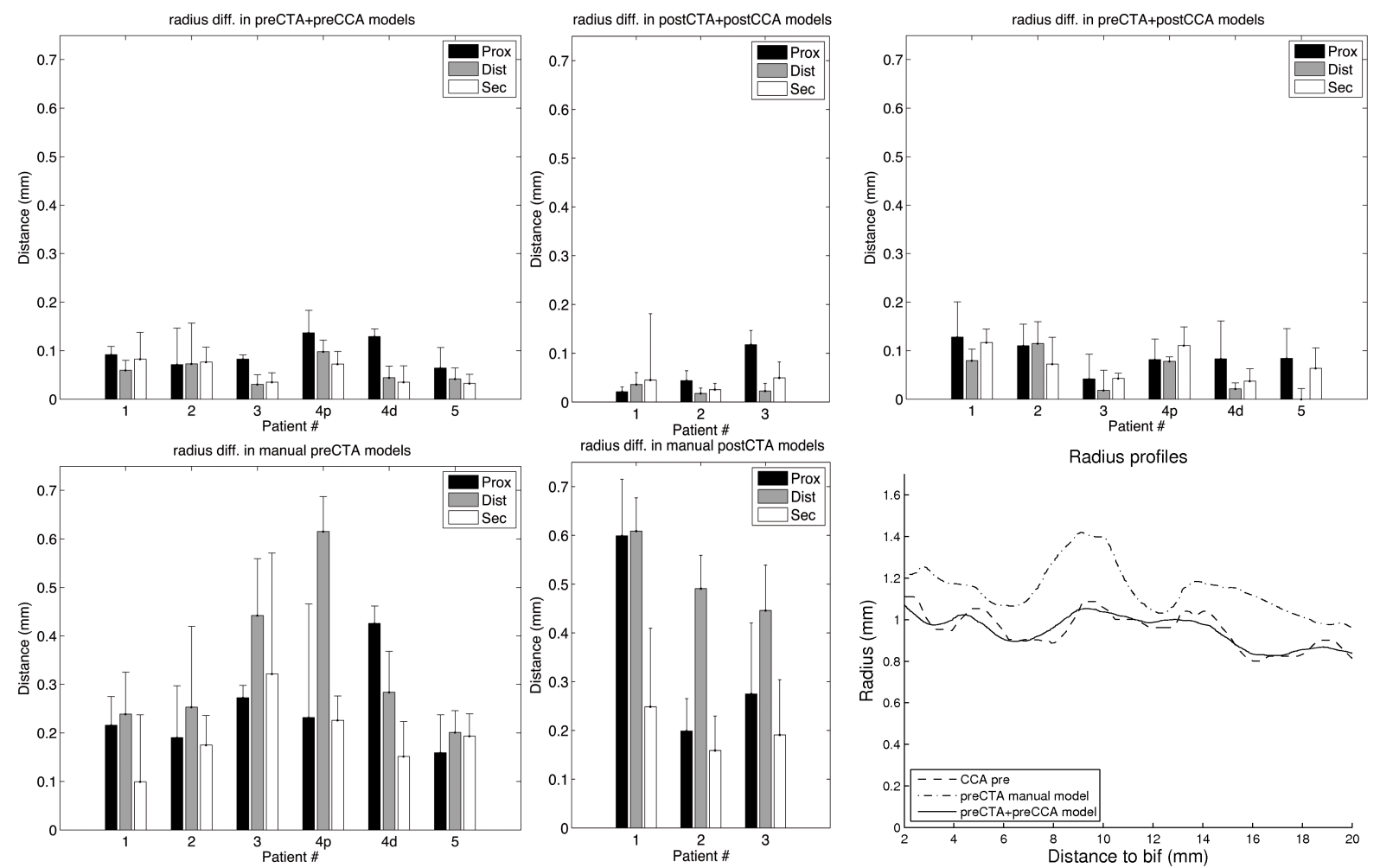

FIG. 9. Average absolute differences, in $\mathrm{mm}$, of the measured vessel radius between the 3D models and the CCA images in all patients, with the std-dev shown as thin lines over the bars. Top row: semi-automatic CTA+CCA models; bottom row: manual models from CTA. First column: preprocedure models (preCTA+preCCA and manual preCTA); second column: post-procedure models (postCTA+postCCA and manual postCTA), third column (top): predictive post-procedure models (preCTA+postCCA). Bottom right: radii values vs distance from the bifurcation center, for one bifurcation branch extracted from a preCTA manual model (blue) a preCTA+preCCA model (red) and from CCA (black). 
TABLE IV. Avarage radii values of vessels measured in CCA data along the vessel centerlines in $\mathrm{mm}$.

\begin{tabular}{c|ccc|ccc} 
& & \multicolumn{3}{|c|}{ Pre-procedure } & \multicolumn{3}{|c}{ Post-procedure } \\
Patient \# & Prox & Dist & Sec & Prox & Dist & Sec \\
\hline \hline 1 & 1.436 & 1.604 & 1.165 & 2.155 & 2.120 & 1.385 \\
2 & 1.684 & 1.078 & 0.924 & 1.906 & 1.482 & 0.906 \\
3 & 1.197 & 0.968 & 0.710 & 1.506 & 1.294 & 0.925 \\
$4 \mathrm{p}$ & 1.561 & 0.791 & 0.942 & 1.603 & 1.430 & 0.962 \\
$4 \mathrm{~d}$ & 0.791 & 0.975 & 0.568 & 1.430 & 1.036 & 0.654 \\
5 & 1.434 & 0.910 & 0.949 & 1.702 & 1.609 & 1.073 \\
\hline
\end{tabular}

\section{E. Murray's law analysis}

The hemodynamic and geometry of vessel bifurcations have been previously analyzed in the literature, and based on a minimum energy hypothesis, vascular trees are known to obey 360 the Murray's law ${ }^{25,28}$, that states that for a given vessel branch and its corresponding vessel daughters, their radii obey this relationship:

$$
r_{\text {prox }}^{3}=r_{\text {dist }}^{3}+r_{\text {sec }}^{3}
$$

with $r_{\text {prox }}$, the radius of the parent vessel, that we call proximal branch, and $r_{d i s t}, r_{s e c}$, the radii of the daughter vessels, that we call distal and secondary branches. In this section, we analyze the Murray's ratio (MR) defined as:

$$
\mathrm{MR}=\frac{r_{p r o x}^{3}}{r_{\text {dist }}^{3}+r_{\text {sec }}^{3}}
$$

Deviation of this value from 1, will give us information about abnormal geometry of a given bifurcation. This analysis is of special interest because non-compliance of Murray's law is expected to be associated with changes in wall shear stress along the bifurcation walls, and therefore, to a higher degree of calcification at bifurcations ${ }^{9}$. Then, from the radii values already computed in our models, we have measured the MR of each patient, for all models, 370 and for CCA data, plotting them in Fig. 10. It can be seen from this figure that all patients, except patient \#4 (more distal bifurcation treated) improved their MR according to our 
models (black bars) and to the CCA data (white bars). However, according to the manual models generated using CTA alone (grey bars), there are some large discrepancies, specially for the post-procedure MR of patient \#2, and pre-procedure MR of patient \#4 (more distal 375 bifurcation treated). Deviations in patient \#2 are mostly due to an underestimation of the distal branch radius in the manual postCTA model, compared to our models and CCA alone. For the patient \#4 (more distal branch), differences are due to overestimation in the proximal branch and underestimation in the two other branches in the manual preCTA model.
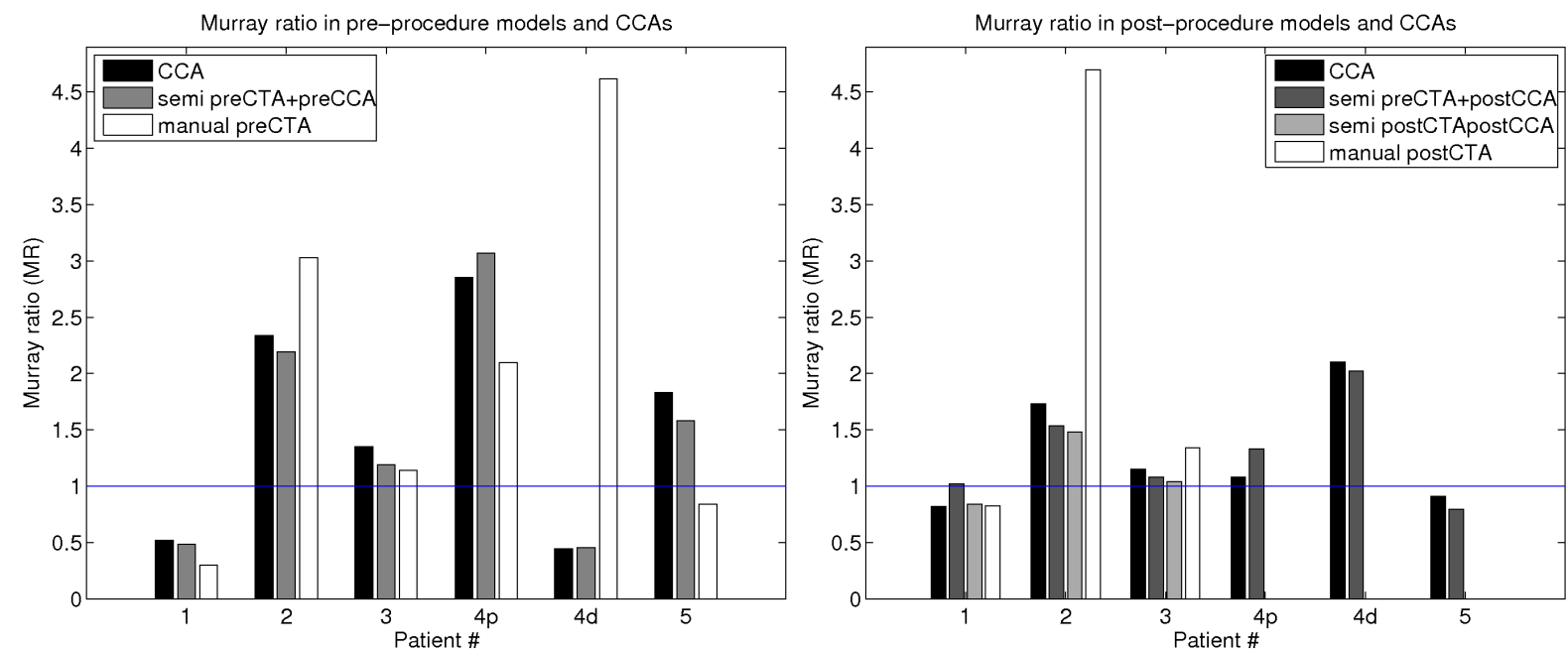

FIG. 10. Murray ratio for each bifurcation treated, computed using semi-automatic CTA+CCA models, using manual CTA models and CCA images, in the pre (left) and post-procedure cases (right).

\section{${ }_{380}$ IV. DISCUSSION}

As shown in table III and Fig.7, models obtained using preCTA+preCCA data are similar numerically and visually to manual models generated with preCTA data, where the overlap between them gives a DS index of 0.79 in average and average distance of $0.279 \mathrm{~mm}$. Similar values are found between semi-automatic postCTA+postCCA models and manual models 385 using postCTA with DS index of 0.764 and average distance of $0.366 \mathrm{~mm}$. These values are indicators of good agreement, but they also evidence differences, as a slight overestimation of the lumen of CTA models, especially in stenosed regions. 
To decide which models are more reliable, we compared them using the radii extracted from CCA data as the reference. In this case, the radii obtained from the models generated 390 using both modalities were clearly more similar to the reference CCA, with average radius difference of $0.06 \mathrm{~mm}$, while in CTA manual models, the average radius difference was of $0.30 \mathrm{~mm}$. These results provide enough evidence to decide that models from our fusion of modalities are more reliable.

The main reason why the differences between the models obtained with our method 395 are better than models generated using CTA information only, is the inclusion of CCA information. CCA images allows more accurate radius estimation than CTA due to better resolution and image contrast and lower noise. The experiments performed indicate that the mapping of the estimated radii can be considered good in a small neighborhood around the bifurcation, due to our hypothesis of planar correspondence between the projection used 400 and the plane formed by the bifurcation.

With respect to the predictive post-procedure models using preCTA+postCCA our results showed mild agreement with manual models obtained with postCTA, having in average DS indices around 0.1 less than postCTA+postCCA models. This is mainly due to centerline misalignment, and centerline changes after PCI. Therefore, our predictive models do not 405 tackle these geometric changes, reducing slightly the overlap with respect to post-procedure CTA centerlines. In our opinion, the changes observed are small in terms of vessel curvature and bifurcation angles after PCI.

These predictive models show good agreement with respect to postCCA, which opens the possibility to have good approximations of post-procedure models right after patient 410 intervention, without the need of waiting for an additional CTA scanner. As shown by the Murray law analysis performed here, geometric changes can be captured with our generated models with the same accuracy as CCA, and therefore, these 3D models can be used to perform patient specific hemodynamic simulations that allow to study the risk of restenosis and thrombosis.

Finally, experiments performed using a phantom with geometry and image characteristics comparable to real cases, showed that the models obtained are very close to the ground truth with average distance values around $0.1 \mathrm{~mm}$. The effect produced in the models when changing the acquisition angle in CCA was low, up to $0.12 \mathrm{~mm}$ of difference from the ground truth in average and $0.42 \mathrm{~mm}$ of maximum distance, when the deviation reached 15 degrees 
420 from the parallel position.

\section{CONCLUSIONS}

We have proposed a method for realistic modeling of coronary bifurcations combining information from two modalities: CTA and CCA. We have shown the advantages of using both modalities together: from CTA, the 3D vessel trajectories and the visible atherosclerotic ${ }_{425}$ plaque are used, and from CCA we obtained a more accurate estimation of the lumen radius, before and after stenting. The results obtained from a cardiac phantom were successful, showing low distances to the ground truth. For real patients, the results obtained were also satisfactory, and the models obtained were in agreement with both the CTA and the CCA data. Numerical comparison with manual segmentation on CTA images showed a good 430 accuracy of our models, and the comparison of the radii values with respect to CCA images, showed that this method improves the accuracy with respect to CTA used alone. Therefore, it has been shown qualitatively and quantitatively, that the assumptions made are valid, at least with the data sets used here, and therefore this method shows it to be a good technique to obtain realistic results using images acquired during normal clinical practice, before and 435 after PCI. From the results obtained, we believe that the low computational cost required (in the order of 5 minutes or less), with a minimal user interaction, is worth it for the gain in accuracy obtained, reducing the radius differences with respect to CCA data from 0.30 $\mathrm{mm}$ using only CTA, to $0.06 \mathrm{~mm}$ using both modalities, in average.

From the accurate models obtained here, geometric simulations can be performed (stent 440 deployment or vessel wall deformation simulations), which can be extremely useful to assess geometrically the outcome of endovascular interventions. On the other hand, hemodynamic simulations can be carried out only if accurate surface models are available, allowing one to study in detail the blood flow and therefore, to be able to detect possible risk of restenosis and thrombosis, by looking at abnormal values of wall shear stress ${ }^{29}$.

\section{${ }_{445}$ Acknowledgements.}

This work was supported by the Spanish Industrial and Technological Development Center (cvREMOD CEN-20091044), R.C. is funded by a Beatriu de Pinós fellowship from 
AGAUR. The authors want to thank Dr. Marta Sitges, Dr. T. M. De Caralt, Dr. N.

Solanes, Dr. M. Rigol, and D. Flores from Hospital Clinic, Barcelona, for their valuable ${ }_{450}$ support especially during the image acquisitions.

\section{REFERENCES}

${ }^{1}$ Louvard, Y., Lefevre, T., Morice, M.c.: Percutaneous Coronary Intervention for Bifurcation Coronary Disease. Heart 90 (2004) 713-722

${ }^{2}$ Sharma, S.K., Sweeny, J., Kini, A.S.: Coronary Bifurcation Lesions : A Current Update.

${ }^{3}$ Iakovou, I., Ge, L., Colombo, A.: Contemporary Stent Treatment of Coronary Bifurcations. J. Am. Coll. Cardiol. 46(8) (2005) 1446-1455

${ }^{4}$ Movahed, M.R., Kern, K., Thai, H., R, E., Friedman, M., Slepian, M.: Coronary artery bifurcation lesions: a review and update on classification and interventional techniques. 460 Cardiov. Revascularization Med. 9 (2008) 263-268

${ }^{5}$ Louvard, Y., Thomas, M., Dzavik, V., Hildick-smith, D., Galassi, A.R., Pan, M., Burzotta, F., Zelizko, M., Dudek, D., Ludman, P., Sheiban, I., Lassen, J.F.: Classification of Coronary Artery Bifurcation Lesions and Treatments: Time for a Consensus ! Catheter. Cardio. Inte. 183 (2008) 175-183

${ }^{465}{ }^{6}$ Arbab-Zadeh, A., Texter, J., Ostbye, K.: Quantification of lumen stenoses with known dimensions by conventional angiography and CT: Implications for the use of angiography as a gold standard. Heart 96 (2010) 1358-1363

${ }^{7}$ Zimmet, J.M., Miller, J.M.: Coronary Artery CTA : Imaging of Atherosclerosis in the Coronary Arteries and Reporting of Coronary Artery CTA Findings. Tech. Vas. interventional Rad. 9 (2006) 218-226

${ }^{8}$ Mortier, P., Holzapfel, G.A., De Beule, M., Van Loo, D., Taeymans, Y., Segers, P., Verdonck, P., Verhegghe, B.: A Novel Simulation Strategy for Stent Insertion and Deployment in Curved Coronary Bifurcations: Comparison of Three Drug-Eluting Stents. Ann. Biomed. Eng. 38(1) (2010) 88-99

${ }_{475}{ }^{9}$ Schoenenberger, A., Urbanek, N., Toggweiler, S., Seelos, R., Jamshidi, P., Resink, T., Erne, P.: Deviation from murray's law is associated with a higher degree of calcification in coronary bifurcations. Atherosclerosis (2012) 
${ }^{10}$ Wellnhofer, E., Osman, J., Kertzscher, U., Affeld, K., Fleck, E., Goubergrits, L.: Flow simulation studies in coronary arteries - Impact of side-branches. Atherosclerosis 213(2)

480

${ }^{11}$ Lim, M.J., Kern, M.J.: Hemodynamic Rounds Utility of Coronary Physiologic Hemodynamics for Bifurcation, Aorto-Ostial, and Ostial Branch Stenoses to Guide Treatment Decisions. Catheter. Cardio. Inte. 468(February) (2005) 461-468

${ }^{12}$ Schaap, M., Metz, C.T., van Walsum, T., van der Giessen, A.G., Weustink, A.C., Mollet, 485 N.R., Bauer, C., Bogunović, H., Castro, C., Deng, X., Dikici, E., O’Donnell, T., Frenay, M., Friman, O., Hernández Hoyos, M., Kitslaar, P.H., Krissian, K., Kühnel, C., Luengo-Oroz, M.a., Orkisz, M., Smedby, O., Styner, M., Szymczak, A., Tek, H., Wang, C., Warfield, S.K., Zambal, S., Zhang, Y., Krestin, G.P., Niessen, W.J.: Standardized evaluation methodology and reference database for evaluating coronary artery centerline extraction algorithms.

${ }^{13}$ Zhu, H., Warner, J., Gehrig, T., Friedman, M.: Comparison of coronary artery dynamics pre- and post-stenting. J. Biomech. 36 (2003) 689-697

${ }^{14}$ Yang, J., Wang, Y., Liu, Y., Tang, S., Chen, W.: Novel approach for 3-D reconstruction of coronary arteries from 2 uncalibrated angiographic images. IEEE Trans. Image Process. 18(7) (2009) 1563-1572

${ }^{15}$ Blondel, C., Malandain, G., Vaillant, R., Ayache, N.: Reconstruction of coronary arteries from a single rotational X-ray projection sequence. IEEE Trans. Med. Imaging 25(5) (2006) 653-663

${ }^{16}$ Jandt, U., Schfer, D., Grass, M., Rasche, V.: Automatic generation of 3D coronary artery centerlines using rotational X-ray angiography. Med. Imag. Anal. 13(6) (2009) 846-858

${ }^{17}$ Cárdenes, R., Díez, J., Larrabide, I., Bogunović, H., Frangi, A.: 3D modeling of coronary artery bifurcations from CTA and conventional coronary angiography. In: T. Peters, G. Fichtinger and A. Martel, (Eds.), MICCAI, LNCS, Springer-Verlag, Part III. Volume 6894. (2011) 395-402

${ }^{505}{ }^{18}$ Aylward, S.R., Bullitt, E.: Initialization, noise, singularities, and scale in height ridge traversal for tubular object centerline extraction. IEEE Trans. Med. Imaging 21 (Feb. 2002) 61-75

${ }^{19}$ Aylward, S., Pace, D., Enquobahrie, A., McCormick, M., Mullins, C., Goodlett, C., Reynolds, P.,: TubeTK, segmentation, registration, and analysis of tubular structures 
${ }^{20}$ Manniesing, R., Viergever, M.A., Niessen, W.J.: Vessel enhancing diffusion: A scale space representation of vessel structures. Med. Image Anal. 10(6) (2006) 815-825

${ }^{21}$ Mueller, D., Maeder, A.: Robust semi-automated path extraction for visualising stenosis of the coronary arteries. Comput. Med. Imag. Grap. 32 (2008) 463-475

${ }_{515}{ }^{22}$ Hassouna, M.S., Farag, A.A.: Variational curve skeletons using gradient vector flow. IEEE Trans. Pattern Anal. 31(12) (Dec. 2009) 2257-2274

${ }^{23}$ Frangi, A., Niessen, W., K.L. Vincken, M.V.: Multiscale vessel enhancement filtering. In: W.M. Wells, A. Colchester and S.L. Delp (eds.), MICCAI'98, LNCS, Berlin, Germany. Volume 1496. (1998) 130-137

${ }_{520}^{24}{ }^{24}$ akakura, K., Yasu, T., Kobayashi, Y., Katayama, T., Sugawara, Y., Funayama, H., Takagi, Y., Ikeda, N., Ishida, T., Tsuruya, Y., Kubo, N., Saito, M.: Noninvasive tissue characterization of coronary arterial plaque by 16-slice CT in acute coronary syndrome. Angiology 52(2) (2006) 155-160

${ }^{25}$ Fung, Y.: Biomechanics. Circulation. Springer, New York, 2nd edn. (1997) ${ }_{525}^{26}$ Yushkevich, P.A., Piven, J., Cody Hazlett, H., Gimpel Smith, R., Ho, S., Gee, J.C., Gerig, G.: User-guided 3D active contour segmentation of anatomical structures: Significantly improved efficiency and reliability. Neuroimage 31(3) (2006) 1116-1128

${ }^{27}$ Sorensen, T. A method of establishing groups of equal amplitude in plant sociology based on similarity of species and its application to analyses of the vegetation on Danish commons. Biologiske Skrifter, Kongelige Danske Videnskabernes Selskab, 5(4) (1957) 1-34

${ }^{28}$ Murray, C.: The physiological principle of minimum work applied to the angle of branching of arteries. J. Gen. Physiol. 9 (1926) 835-841

${ }^{29}$ Samady, H., Eshtehardi, P., McDaniel, M.C., Suo, J., Dhawan, S.S., Maynard, C., Timmins, L.H., Quyyumi, A.A., Giddens, D.P.: Coronary artery wall shear stress is associated with progression and transformation of atherosclerotic plaque and arterial remodeling in patients with coronary artery disease. Circulation. 124(7) (2011) 779-788 